\title{
Three Stages of Bonding Mode before Nucleation of Metal Crystals
}

\author{
Hiroshi Fujita \\ Emeritus Proffesor of Osaka University, Home address: Yamatedai 5-12-22, Ibaraki 567-0009, Japan
}

\begin{abstract}
Effect of particle size on atomistic structures of vapor deposited Au-atom clusters has been examined by electron microscopy with the following results: (a) Icosahedrons are first formed, and they grow by two types of face sharing of further icosahedrons. (b) The lattice constant decreases more than about $1 \%$ in transition from icosahedral structures to cuboctahedral ones, which are a fcc-type structure, when average size of atom clusters becomes larger than about $\Phi 3 \mathrm{~nm}$. (c) Embryo of the final fcc structure is formed when the size of atom clusters increases to about $\Phi 8 \mathrm{~nm}$, and the lattice constant considerably increases more than $2 \%$ in spite of the same fcc structure as cuboctahedral ones. After that, the lattice constant scarcely changes even when the particle size increases more than $\Phi 20 \mathrm{~nm}$.

These facts are considered to be closely related to heterogeneous distribution of electric charge within atom clusters. Based on the results, relationships between electronic structures in the three stages and anomalous behavior of metal-atom clusters, consisting of both fcc-type and bcc-type elements, are discussed. [doi:10.2320/matertrans.MRA2006635]
\end{abstract}

(Received December 12, 2006; Accepted March 19, 2007; Published May 25, 2007)

Keywords: atom cluster, icosahedron, cuboctahedron, $\delta$-phase, embryo, electron spin

\section{Introduction}

Metal atoms have two typical electronic structures, i.e., the ground state of each isolated atom and the bonding state of the corresponding crystal. The former changes to the latter as the number of constituent atoms increases, and the Engels notation $^{1)}$ is frequently used for the latter, as used in the present report. Furthermore, the atomic bonding among constituent atoms results from interaction between electrons and ion cores of the atoms, and in metals, especially transition metals, all electrons outside of those in the corresponding inert gas atoms contribute to the atomic bonding by the following two types: (a) Delocalized electrons: They easily move all over materials like free electrons by using vacant orbitals. Electrons of the outermost orbital generally belong to this type. (b) Localized electrons: They are localized in bonding parts between neighboring atoms so that atomistic structure is decided. They are sensitive not only to the number of constituent atoms but also kind of elements, as mentioned later, and make resonance among neighboring bonding parts.

Recently an attention has been paid on nanometer sized materials because of their anomalous behavior, such as remarkable decrease of melting temperature, ${ }^{2,3)}$ spontaneous mixing of guest-atoms, ${ }^{4,5)}$ easy shape change, ${ }^{6}$ rapid coalescence like liquid drops ${ }^{7)}$ etc. The author has reported ${ }^{8-13)}$ that when the size of Au-particles becomes smaller than the nucleus of crystal their behavior anomalously changes, and that the following three stages of atomistic structure occur before the nucleation of fcc crystals; Formation of (1) icosahedral structures, (2) cuboctahedral structures of a fcctype, and (3) embryo of the final crystal. Here, these nanometer sized materials are named "atom clusters".

In order to clarify the anomalous behavior of atom clusters, detail information about both their atomistic and electronic structures, especially on the bonding mode, is necessary in each of these stages. In the present report, the size effect on the atomistic structure is examined by the electron microscopy on Au-atom clusters which are vapor deposited onto amorphous carbon films kept at about $300 \mathrm{~K}$. Based on the experimental results, the effect of atomistic and electronic structures on the anomalous behavior in the three stages are discussed on metal-atom clusters of both fcc-type and bcctype transition elements.

\section{Experimental Results}

Most of metallic elements are considered to form a close packed structure such as icosahedron at the beginning of formation of their atom clusters, ${ }^{14)}$ and nucleation of the corresponding crystals is considered to occur through aforementioned intermediate stages. ${ }^{10-12)}$

Each icosahedron generally has 12 coordination number around the center atom, in which interatomic distance between adjacent peripheral atoms is larger than that between the center atom and each peripheral one. ${ }^{12)}$ The latter corresponds to a radius of a sphere, hereafter I-sphere. Therefore, each peripheral atom can move on the surface of Isphere, and thus each icosahedron transforms to a cuboctahedron without any thermal diffusion when constituent atoms increases in the number so that the electric charge is homogenized as a whole to reduce the difference in interatomic distances. ${ }^{12)}$

Electron microscope images do not show straight lattice fringes in the first stage when a Au-atom cluster is less than about $\Phi 3 \mathrm{~nm} .{ }^{12)}$ When cluster size becomes larger than about $\Phi 3 \mathrm{~nm}$, which belongs to the second stage, multiple straight lattice fringes showing a fcc-type structure appear. At the beginning of this stage, however, contrast of peripheral regions of these clusters is not sharp, and takes a round shape without any facet structure. ${ }^{10)}$ Here, it is noted that both icosahedral and cuboctahedral structures always make spontaneous mixing of guest-atoms into host-atom clusters even at lower temperatures below $300 \mathrm{~K}$, and that this anomalous phenomenon is continued until the nucleation is finished, i.e., up to about $\Phi 15 \mathrm{~nm}$ in isolated Au-atom clusters. ${ }^{4,10)}$ This phenomenon is also sensitive not only to atomic size ratio but also to electronegativity difference between host- and guest-atoms, especially to the latter. ${ }^{5-11)}$ This suggests that the electric charge is distributed heterogeneously inside these atom clusters even in cuboctahedral structures. $^{10)}$ 


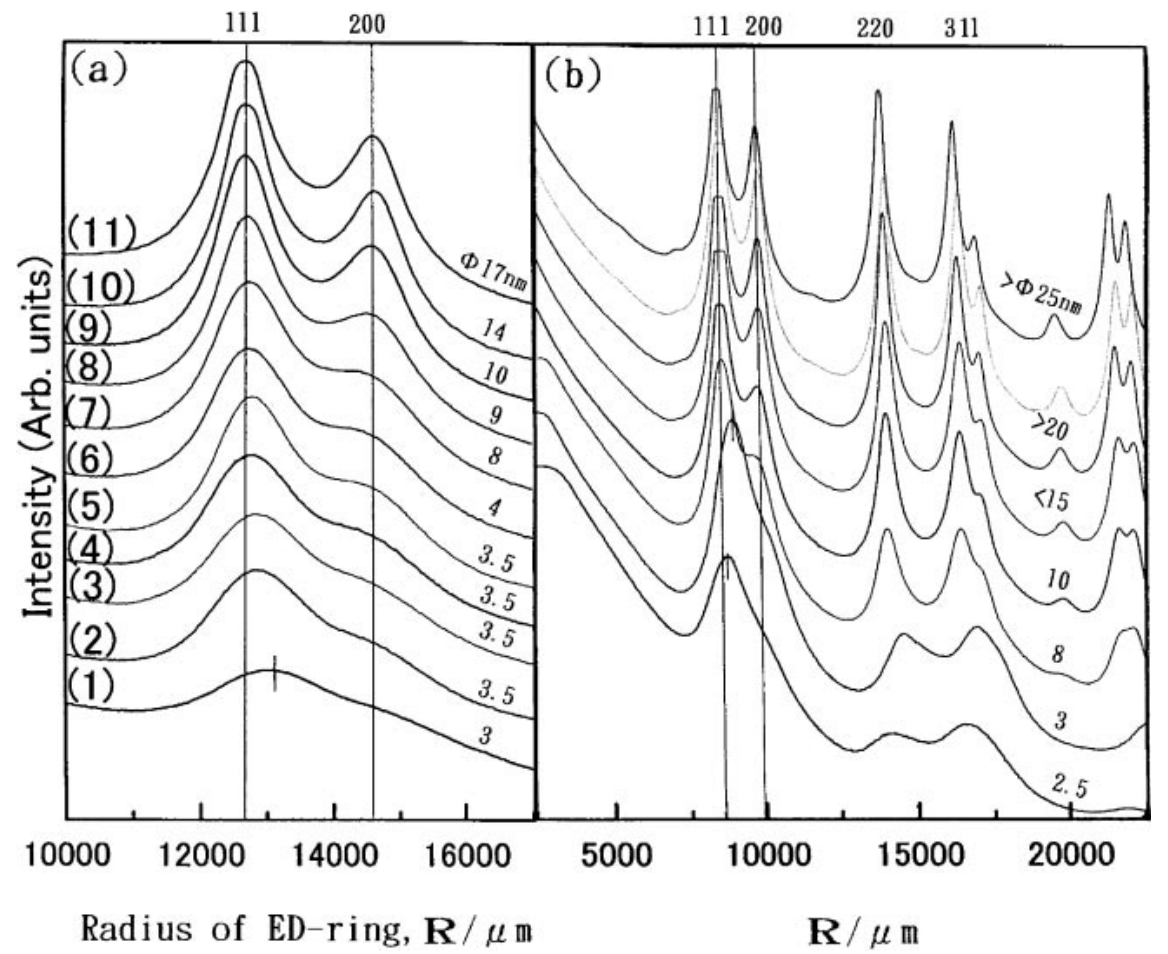

Fig. 1 Size effect on electron diffraction (ED) rings of isolated Au-atom clusters. Curves (a) and (b) show intensity profiles versus radii(R) of ED-rings in each of different series of the specimens, in which curve (a) was taken at a high magnification of ED-rings. Estimated average size of particles is shown on each curve, respectively.
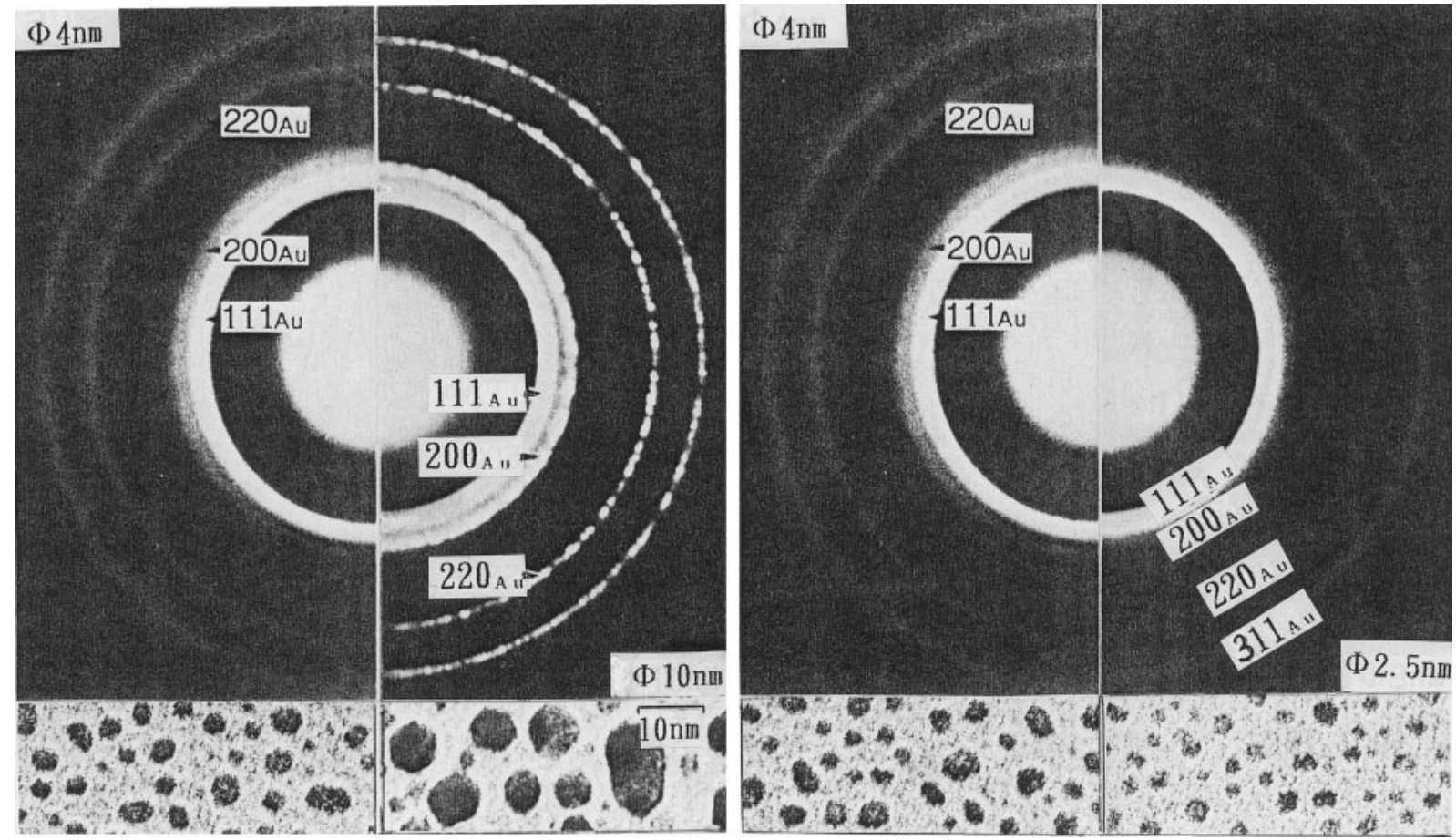

Fig. 2 Average size and the corresponding ED-patterns at different stages in Au-atom clusters. The specimens used are different from those of Fig. 1, and atom clusters of $\Phi 2.5,4$ and $10 \mathrm{~nm}$ in average size correspond to icosahedral structures, cuboctahedral structures and after formation of embryo, respectively.

Figures 1 and 2 show the size effect on electron diffraction(ED) rings. Here, profiles and ED-rings in Figs. 1(a) and (b) were taken by imaging plate method, and relative setting error of the center of ED rings by this method is estimated from Fig. 1 to be less about $0.5 \%$ in the lattice constant. In the imaging plate method, magnification of ED-rings is selected depending on the purpose, and then the center of ED-rings is set on a fixed position. After that, radii(R) and intensity profiles of ED-rings are measured. Furthermore, the same size of ultrafine particles is generally difficult to obtain, and thus average size must be used practically as the particle size. This is a reason why, in the present work three series of 


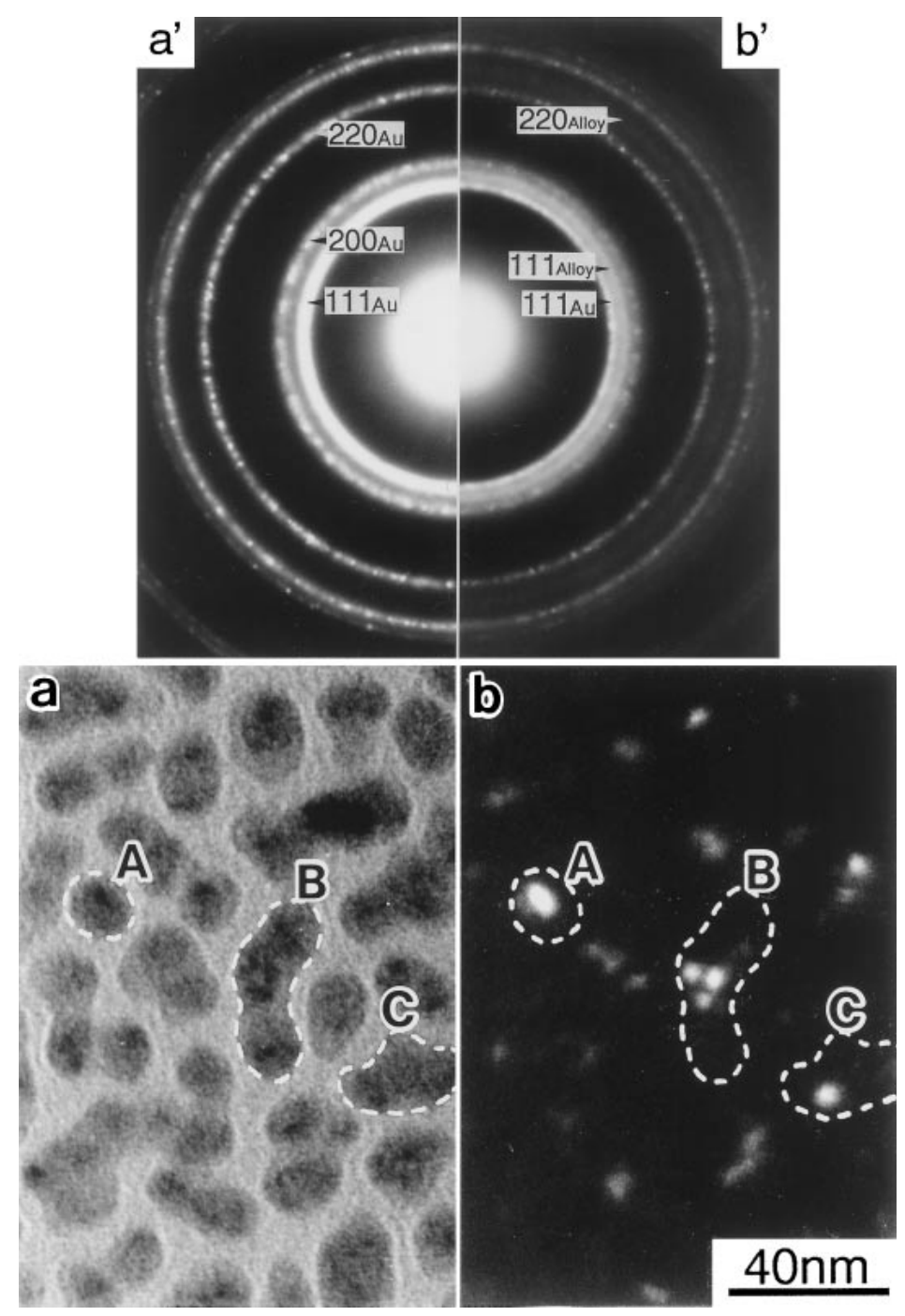

Fig. 3 Formation of embryos at the center region of Au-atom clusters. Micrographs (a) and (b) are a bright field image and a dark field one taken with $\{111\}$ reflection of Au-atom clusters. ED-rings $\left(\mathrm{a}^{\prime}\right)$ shows pure Au-atom clusters themselves, and $\left(\mathrm{b}^{\prime}\right)$ of Au-Cu alloys taken after apontaneous mixing of $\mathrm{Cu}$-atoms into Au-atom clusters. Bright particles in micrograph (b) show embryos of pure Au-blocks, in which spontaneous mixing of $\mathrm{Cu}$-atoms hardly occurs. Contrarily, $\mathrm{Cu}$-atoms were spontaneously mixed into Au-atom clusters in surrounding dark regions taking cuboctahedrtal structures. (Courtesy of Prof. H. Mori).

vapor deposited Au-particles were used to show accuracy of these estimated average sizes, e.g., as seen in curves $(2) \sim(5)$ showing the same $\Phi 3.5 \mathrm{~nm}$ in Fig. 1(a). A low magnification was used in Fig. 1(b) to show a wide range of ED-rings, in which both ED-rings of atom clustrers of about $\Phi 2.5 \mathrm{~nm}$ and those of larger sizes up to about $\Phi 25 \mathrm{~nm}$ are shown. In Fig. 2, size distribution of atom clusterts and the corresponding EDrings are shown at each stage, corresponding to icosahedral structures, cuboctahedral ones and after formation of embryo of the final crystals.

In Fig. 1, change of the lattice constant clearly appears when the size becomes larger than not only at about $\Phi 3 \mathrm{~nm}$ but also at about $\Phi 8 \mathrm{~nm}$. The first change corresponds to transition from icosahedral structures to the cuboctahedral ones, and the lattice constant corresponding to a fcc-type structure decreases more than about $1 \%$. The second one occurs when the embryo of Au-crystals is formed within each cuboctahedral structure, and then the lattice constant increases more than about $2 \%$ even though both atomistic structures belong to the same fcc one.
Furthermore, ED-rings corresponding to $\{200\},\{220\}$ and so on in a fcc-type structure are not so clear even in the cuboctahedral structures of $\Phi 4 \mathrm{~nm}$ in average size, and scarcely appear in icosahedral structures, as seen in Figs. 1 and 2 . This suggests that crystallographic planes corresponding to higher orders of diffraction do not develop sufficiently within atom clusters of these sizes. After the embryo of the final crystal is formed at the center region of each atom cluster, ${ }^{10)}$ all ED-rings sharply appear, as in Figs. 1 and 2.

It is also noted in Fig. 1 that the half value width of intensity profiles of ED-rings decreases, but that each peak position of rings scarcely changes after the size of atom clusters becomes larger than $\Phi 8 \mathrm{~nm}$, at which the embryo is formed, up to more than $\Phi 25 \mathrm{~nm}$, which is larger than nucleus size of about $\Phi 15 \mathrm{~nm}$. Namely, the peak positions slightly increase in the lattice constant, i.e., less than about $0.5 \%$ when the embryo grows to about $\Phi 10 \mathrm{~nm}$, but hardly change after that.

Figure 3 was taken after spontaneous mixing of $\mathrm{Cu}$-atoms into $\mathrm{Au}$-atom clusters in which coalescence already occurred 


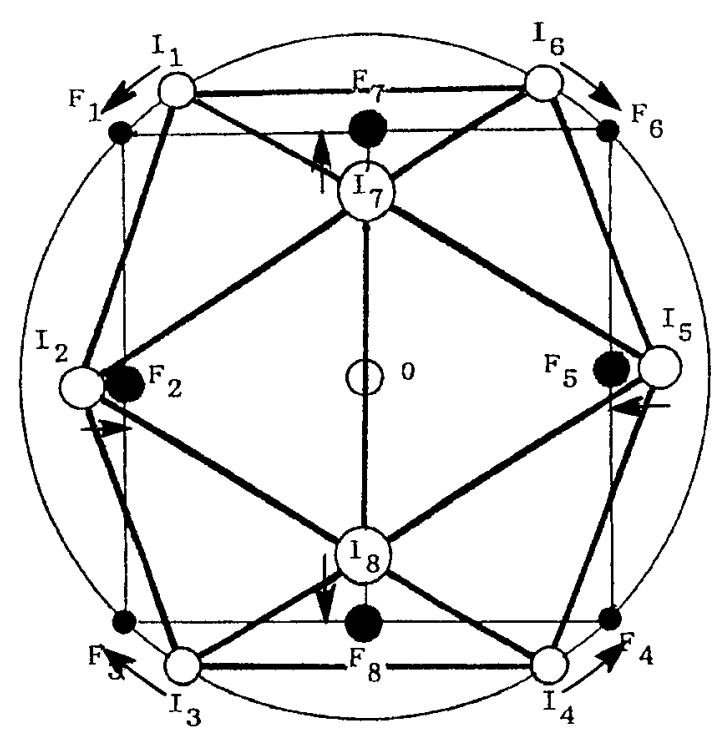

Fig. 4 Transition from an icosahedron (open circles) to a cuboctahedron (closed circles). Movement of consitiuent atoms of the former occurs without any thermal diffusion on the surface of I-sphere, as shown by arrows, as the number of constituent atoms increases.

among many Au-atom clusters. Figure 3(a) is a bright field image, and (b) is a dark field one taken by $\{111\} \mathrm{Au}-$ reflection respectively. It is noted in Fig. 3(b) that an embryo showing a pure Au single block, bright region, is formed within each solid solution of $\mathrm{Au}-(55-65)$ at $\% \mathrm{Cu}$, dark regions, as recognized from the corresponding ED-pattern $\left(b^{\prime}\right)$ at the upper part of Fig. 3. This means that the bonding mode within the embryo is changed from that of the surrounding cuboctahedral structures so that spontaneous mixing of $\mathrm{Cu}$-atoms does not occus. ${ }^{9)}$

It is concluded in Fig. 1 that after formation of the embryo, each position of the intensity peaks is mainly determined with atomistic structure of the embryo, and the half value of intensity profiles results from mixing of the embryo and surrounding cuboctahedral structures until nucleation of crystals is finished. Furthermore, the changes of the lattice constant in Figs. 1 3 show occurrence of changing the bonding mode. The transition from the first stage to the second one is reasonably understood to be due to decreasing the lattice constant, as recognized in Fig. 4. However, the considerably large difference in the transition from the second stage to the third one can not be recognized by such current effects as surface tension, surface structures etc., as discussed in the next section.

\section{Discussions}

\subsection{Surface effects on atomistic structure of atom clusters}

It has been considered that a compression stress denoted by $2 \gamma_{\mathrm{s}} / \mathrm{r}$, $\left(\gamma_{\mathrm{s}}\right.$ : the surface tension, $\mathrm{r}$ : a radius of an ultrafine particle) acts to the center region of the particle. As mentioned above, however, the lattice constant scarcely changes after the embryo is formed at $\Phi 8 \mathrm{~nm}$, even when the value of $r$ increases more than twice. Generally, the nucleus size of crystals $\left(d_{M}\right)$ is a function of the surface energy $\gamma_{s}$ itself, but the value of $d_{M}$ becomes about $\Phi 5 \mathrm{~nm}$ in embedded atom clusters whose $\gamma_{\mathrm{s}}$ is smaller than a half of isolated ones whose $d_{M}$ is about $\Phi 15 \mathrm{~nm}$. Furthermore, the specific surface energy is generally decreases by broad distribution within an atom cluster as cluster size decreases. ${ }^{12}$ ) These are just the reverse results against the effect of surface tension mentioned above.

On the other hand, electron microscope images ${ }^{10)}$ show that the value of $\gamma_{\mathrm{s}}$ decreases considerably in a size range smaller than about $\Phi 5 \mathrm{~nm}$ showing cuboctahedral structures already. When the size becomes larger than $\Phi 5 \mathrm{~nm}$, such surface structures as facet ones also appear. These surface structures also induce the shear stress which is inversely proportional to the distance from the surface, i.e., the interatomic distance is mostly affected at the top surface, but actually the transition begins at the center region. Remarkable decrease of melting point of atom clusters also shows decreasing $\gamma_{\mathrm{s}}$ because of increasing the homologous one of room temperature.

The facts mentioned above show that more than $2 \%$ difference in the lattice constant can not be understood by the current surface effects. Here, two body potential among atoms in a many body system is considered to consist of the following three damped oscillations of (a) short, (b) intermediate and (c) long distances, respectively. ${ }^{15)}$ The atomistic structure is sensitive to these three terms, especially to the last term(c) which results from overlapping degree among ion core potentials. Therefore, the large change of the lattice constant in the third stage is considered to depend on such different reasons as heterogeneous distribution of electric charge, as mentioned below.

\subsection{Formation of icosahedral structures}

It has been shown experimentally that an icosahedron and icosahedral structures are formed at the beginning of formation of Au-atom clusters. ${ }^{12,16,17)}$ This means that the icosahedron is more stable than a fcc-type structure at this stage. Since the icosahedron has the characteristics mentioned already, the bonding potential among constituent atoms in an icosahedron can be expressed by the LennardJones potential or something like this, because kinetic energy of this stage is considerably large even at about $300 \mathrm{~K}$.

Now, difference in interatomic distance between peripheral atoms and the radius of I-sphere results from difference of electric charge in both ones, because electron density considerably increases around the center atom. ${ }^{12)}$ Here, the radius of I-sphere is assumed to be due to decreasing apparent size of the center atom, an icosahedron becomes more stable than a fcc structure when the apparent radius becomes smaller about $3 \%$ than that of peripheral atoms. ${ }^{12}$ )

Since peripheral atoms on the surface of I-sphere are widely separated, further constituent atoms will deposited on sites above the center of these expanded triangles, respectively. As a result, the original icosahedron grows up to icosahedral structures which consist of face and/or edge sharing of additional icosahedrons. ${ }^{13)}$ Here, there are two types of the face sharing of icosahedrons onto the original one, as shown in Figs. 5(a) and (b).

Figures 5(a) and (b) show each of bcc-type and 5-domainstype face sharing respectively, in which additional atoms are considered to deposit first onto triangles, e.g., such shaded 
(a)

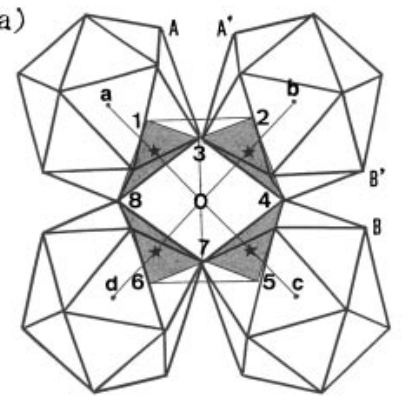

(b)

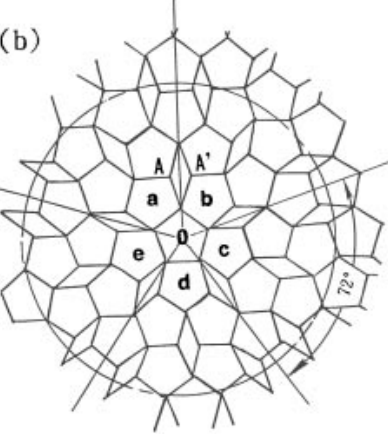

(a)

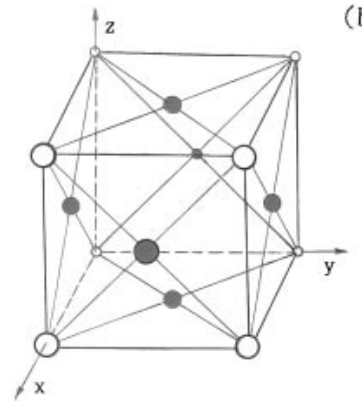

(b)

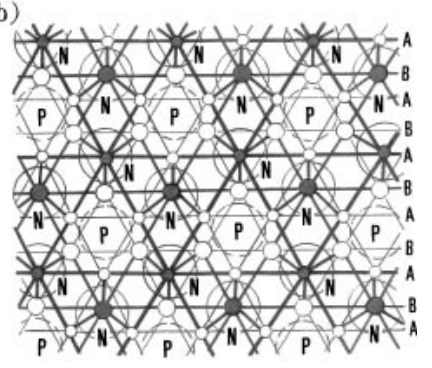

Fig. 5 Two types of face sharing of icosahedrons in atom clusters of fcctype elements. Figures (a) and (b) show bcc-type face sharing and 5domains-type one respectively. The former results in a single domain of cuboctahedral structure and the latter in a multiple-twinned one.

ones as $\Delta 138, \Delta 234$ etc. in Fig. 5(a), of the original icosahedron under the Lennard-Jones potential. And thus, these deposited atoms will take such symmetry positions as a, $\mathrm{b}, \mathrm{c}, \cdots$ against the center atom(o) of the original icosahedron through each triangle, as shown in Figs. 5(a) and (b). Around these additional atoms new icosahedrons are successively formed by repeating the same process, as shown in Figs. 5(b). Here, a triangle in Fig. 5(a) becomes parallel to substrate surface, but gaps among icosahedrons increases compared with that in Fig. 5(b). And thus, 5-domains-type ones are preferentially formed in general. During growth process of an icosahedral structure electric charge is gradually homogeneized from the center region because of homogenizing coordination number of each constituent atom, while many gaps are still accompanied, as seen in Figs. 5(a) and (b).

In both types, constituent atoms are supplied from both the upper vapor source and bottom substrate surface. In this process, constituent atoms can not be deposited onto neighboring triangles jointed with an edge, because their interatomic distance becomes larger than that between their centers of gravity. Furthermore, some atoms can not take sites such as corners $\mathrm{A}$ and $\mathrm{A}^{\prime}, \mathrm{B}$ and $\mathrm{B}^{\prime}$ etc. of additional icosahedrons in Fig. 5 because of the same reason, and then either of them becomes vacant. This fact is necessary to take a regular coordination number of the final crystal, ${ }^{12,13)}$ but becomes also one of the origin of heterogeneous distribution of atomic and electronic structures.

\subsection{Transition from icosahedral structures to cubocta- hedral ones}

Since electric charge density is homogeneized around all constituent atoms as the number of constituent atoms increases by the mechanism mentioned above, transition from icosahedral structures to cuboctahedral ones spontaneously occurs without any thermal diffusion when the difference in radius between the center atom and that of peripheral ones decreases less than about 3\%,10) which corresponds to about $\Phi 3 \mathrm{~nm}$ in the size of an atom cluster, as indicated with a chain circle in Fig. 5(b).

At the beginning of transition to cuboctahedral structures, each of the $\{100\}$-like planes can not take a flat shape but still consists of two triangles, as recognized in Fig. 4. Since the lattice constant decreases in cuboctahedral structures, as

Fig. 6 Distribution of electron spins expected in cuboctahedral structures of atom clusters consisting of fcc-type elements. Electron spins in open and shaded atoms show unlike sign each other in both (a) and (b), and two $\{111\}$ planes denoted by A and B are shown in (b) in which bonding orbitals among unlike sign are shown by thick lines. Since electron spins of unlike sign do not arranged alternately on the $\{111\}$ planes, density of valence electrons increases in $\mathrm{N}$-regions but decreases in P-regions in (b).

shown already, constituent atoms are weakly connected by $(s) \sigma$-bonding each other, because of the radius of Fermi hole $\left(r_{\mathrm{s}}\right)$ is sufficiently decreased with increasing the number of surrounding ion cores. Finally they take the regular sites of not only on the $\{100\}$ planes but also on higher orders of crystallographic planes when the size of atom clusters increases more than about $\Phi 8 \mathrm{~nm}$. Here, bcc-type icosahedral structures change to cuboctahedral ones consisting of a single domain each, and the 5-domains-type ones change to cuboctahedral ones consisting of five twins exactly. The former will also be related to a bcc arrangement of icosahedrons in quasicrystals.

When constituent atoms are connected by $(s) \sigma$-bonding, charge distribution is also affected by electron spin. Here, it is considered that interaction force between electron spins of the same sign, i.e., parallel spins, becomes more important among valence electrons compared with the Coulomb interaction, as expected from the Fermi hole when the structure changes to cuboctahedral ones. In this structures, however, electron spins of unlike sign are not always be arranged alternately, especially on the $\{111\}$ planes, as shown in Fig. 6, and thus density of valence electrons becomes heterogeneous around these parallel electron spin pairs.

Figure 6(b) shows the heterogeneous distribution of $s$ electron spins on two adjacent $\{111\}$ planes denoted by A and B. Since $(s) \sigma$-bonding generally shows a spherical symmetry, something of the Coulomb force newly acts among valence electron $\operatorname{rich}(\mathrm{N})$ and poor $(\mathrm{P})$ regions, and thus cuboctahedral structures result in the following behavior: (a) Enhancement of spontaneous mixing of guset-atoms into host-atom clusters. ${ }^{9)}$ (b) Interatomic distance among constituent atoms is reduced by interaction among $\mathrm{N}$ - and P-regions, while the binding strength of $(s) \sigma$-bonding itself is not so strong. The interaction is also much stronger than those of current surface effect mentioned already. (c) Relative glide between these two $\{111\}$ planes easily occurs, because the number of bonding orbitals between them decreases to a half.

Furthermore, heterogeneous distribution of electric charge also results from local movement of constituent atoms in each cuboctahedron on the surface of I-sphere until the final fcc structure is formed. These heterogeneous distribution of electric charge will increase the total energy of the many 

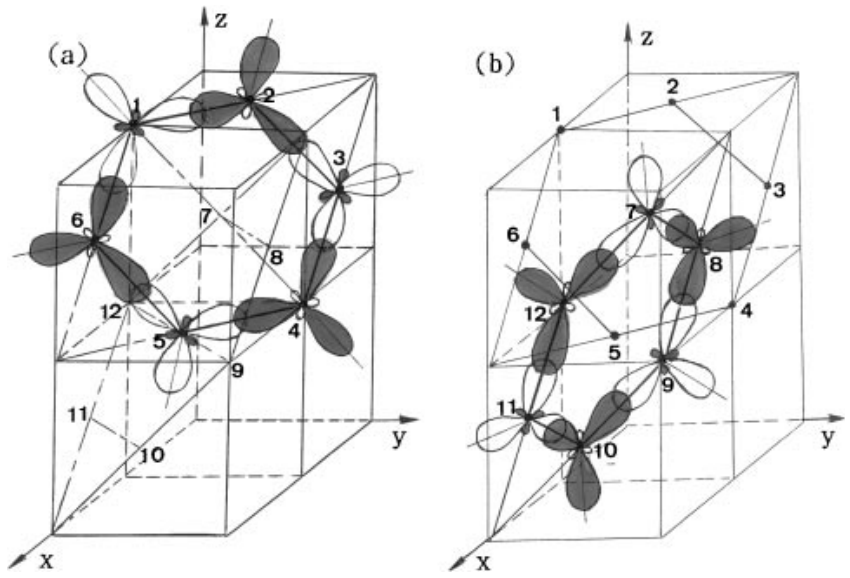

Fig. 7 Distribution of electron spins in a fcc crystals. Electron spins in open and shaded lobes show unlike sign each other. Electron spins of $\left(s p^{2}\right) \sigma$-bond, each of which has three long lobes, are alternately arranged also on all $\{111\}$ planes by mutual resonance, e.g., (111) in (a) and $(1 \overline{1} 1)$ in (b).

body system, and results in the anomalous behavior of atom clusters.

Since even in $\mathrm{Si}$-atom clusters constituent atoms is considered to be increased in the number more than 10 in order to make hybridization of $\left(s p^{3}\right) \sigma$-bonding, ${ }^{18)}$ the final fcc structure does not form in cuboctahedral structures within Au-atom clusters, because the number of Au-atoms is not sufficient.

\subsection{Formation of the embryo within cuboctahedral structures}

The total energy of the second stage is effectively relieved when a suitable hybrid orbital consisting of long lobes is formed, because contribution of the total exchange-correlation energy markedly increases as well as homogenizing the electric charge. Under the condition, the energy band of $s$ electron of constituent atoms sufficiently overlaps with that of the upper $(p)$-orbital in addition to high energy $d$-electrons in their antibonding orbitals nearby. Therefore, the corresponding hybrid orbital, such as $\left(s p^{2}\right)$-orbitals will be formed at the center region of atom clusters. This process successively expands into the surrounding cuboctahedral structures in which expansion of the lattice constant is effectively relieved by easy deformation, especially within isolated atom clusters, as mentioned already. Since $\left(s p^{2}\right) \sigma$-bonding has three long lobes along the $\langle 110\rangle$ directions of a fcc structure, the lattice constant is considered to be considerably increased when the hybrid orbitals are formed, while atomistic structure itself belongs to the same fcc one as that of cuboctahedral structures.

Furthermore, in the case of hybrid orbitals, $\left(s p^{2}\right) \sigma$ bonding, homogeneous distribution of the electric charge results from periodic array of unlike spins by their mutual resonance not only on $\{100\}$ planes but also on all $\{111\}$ planes and others, as shown in Fig. 7. Here, three long lobes of the $\left(s p^{2}\right)$-hybrid orbitals result in a higher binding strength by deep overlapping with those of neighbors compared with that of cuboctahedrtal structures, and thus anomalous behavior of atom clusters hardly occurs after hybridization. ${ }^{12)}$

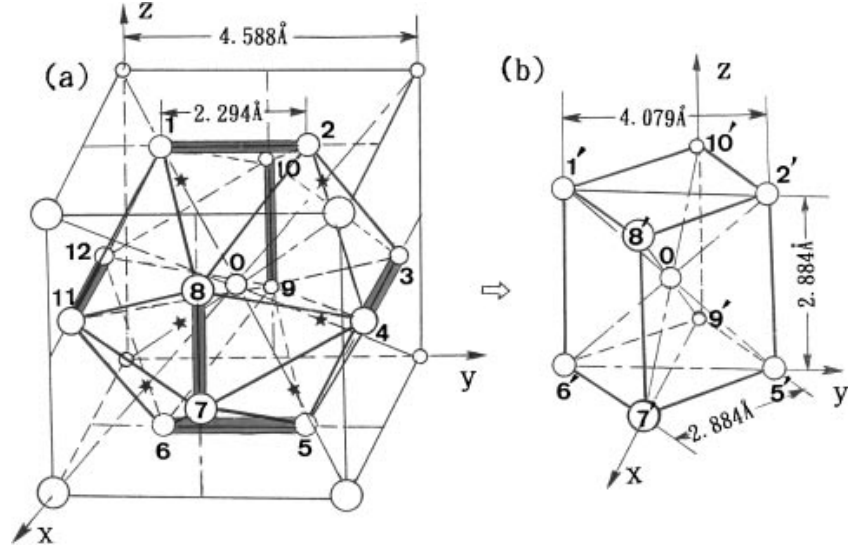

Fig. 8 Formation process of $\delta$-phase of bcc-type elements. Additional atoms in (a) are preferentially deposited onto triangles marked by stars of the first zone of a deformed icosahedron (open circles) on which constituent atoms are most widely separated. Figure (b) shows formation of a unit cell of bcc structure with two pairs of dumbbell-atoms in a unit cell of $\delta$-phase in (a), e.g., each of two pairs are parallel to $\mathrm{y}$ and $\mathrm{z}$ axes respectively in (b).

\subsection{Size effect on atomistic structures of atom clusters of bcc-type elements}

Even in the case of bcc-type elements, icosahedrons are also expected to be formed at the beginning of formation of their atom clusters. Since their icosahedral structures consist of close packed structures, bcc-type elements also will show a fcc-like structure in these ultrafine atom clusters, as suggested by R. Uyeda. ${ }^{18)}$ Furthermore, the three stages of atomistic structure are also expected if $\delta$-phase structure ${ }^{19)}$ is considered to be the second stage, as shown in Fig. 8. Transition of icosahedral structures to $\delta$-phase will occur by the similar way to that in fcc-type elements shown in Fig. 5(a). Here, additional atoms of the second zone are preferentially deposited onto each triangle marked by stars as $\Delta 457, \Delta 359$ etc.of the first zone, on which constituent atoms are most widely separated. As a result, the additional atoms are located at each corner of a cubic structure, as seen in Fig. 8. In Fig. 8(a), however, interatomic distance along the $\langle 100\rangle$ directions is considerably small compared with those of bulk crystals. This is considered to be closely related to a short bonding distance of diatomic molecules by $(s) \sigma-$ bonding. ${ }^{21)}$ Here, antibonding orbitals of $d$-electrons do not exist in $\mathrm{Ti}, \mathrm{V}, \mathrm{Cr}$ etc., and $d$-electrons of bonding orbitals are very close to $s$-electrons in the upper energy level. These facts are closely related to strengthen $(s) \sigma$-bonding, as mentioned above, and also hybrid orbitals along the $\langle 111\rangle$ directions will be formed as constituent atoms increases more in the number. ${ }^{11)}$ During the process, the heterogeneous distribution of electron spins like Fig. 6(b) also results in Fig. 8(a) when the value of $r_{\mathrm{s}}$, which is a function of the number of surrounding ion cores, decreases to a certain value, and finally a bcc structure is formed by the hybridization, i.e., the third stage, as shown in Fig. 8(b). Here, transition between each of different stages is originally carried out without any thermal diffusion of constituent atoms as a result of formation of $(s) \sigma$-bonding. Four atoms in a unit cell, however, must take a considerably long displacement in transition from the second to the third one, i.e., a bcc 
structure. And thus, transitions to the third stage also resuls from formation of the hybrid orbitals, but the process itself will be enhanced by thermal agitation. ${ }^{19)}$

Other types of crystal structure of such metals as $\mathrm{In}^{22}$ are also expected to take the similar three stages of atomistic structure in their atom clusters.

\section{Summary and Conclusions}

The present results are summarized as follows:

(1) There are three stages of bonding mode in Au-atom clusters before nucleation of crystals. Each of them is a function of the number of constituent atoms within atom clusters.

(2) Two-types of icodsahedral structures, i.e., 5-domainstype and bcc-type ones, are formed by face sharing among icosahedrons, in which there are heterogeneous distribution of electric charge. Multiple-twinned cuboctahedral structures result from the former.

(3) Transition from icosahedral structures to cuboctahedral ones occurs when the size increases to more than about $\Phi 3 \mathrm{~nm}$ without any thermal diffusion, by which the lattice constant decreases more than about $1 \%$.

(4) Weak $(s) \sigma$-bonding is formed in cuboctahedral structures, and heterogeneous distribution of electric charge also enhanced within atom clusters not only around parallel electron spins but also by soft-phonon mode of constituent atoms.

(5) The heterogeneous distribution of electric charge in both icosahedral structures and cuboctahedral ones causes anomalous behavior of atom clusters.

(6) The lattice constant considerably increases more than $2 \%$ in the transition from cuboctaheral structures to the final structure, while both of them show the same fcctype structure. This is considered to be due to formation of the hybrid orbitals, e.g., $\left(s p^{2}\right) \sigma$-bond by Engels notation, having long lobes, by which anomalous behavior of atom clusters hardly appear.

(7) Even in other types of metal crystals such as bcc-type elements, the bonding mode within atom clusters is expected to take the similar process before taking the final crystal structures.
The results show the importance of the hybrid orbitals in atomistic structure of metal crystals, as expected from existence of the Burgers vector of dislocations in metal crystals.

\section{Acknowledgements}

A part of this work was supported by "Nanotechnology Support Project of the Ministory of Education, Culture, Sports, Science and Technology (MEXT), Japan at the Research Center for Ultrahigh Voltage Electron Microscopy, Osaka University. The author wishes to express his heartly thanks to Prof. H. Mori for his fruitful discussions and Dr. J. $\mathrm{G}$. Li for his effort in the extensive experimental work.

\section{REFERENCES}

1) N. Engels: Refered by G. C. Pimentel and R. D. Spratley: Chemical bonding clarified through quantum mechanics, Holand-Day, Inc., San Francisco (1969) pp. 278-283.

2) M. Takagi: J. Phys. Soc. Jpn. 9 (1954) 359-363.

3) D. A. Buffet and J. P. Boel: Phys. Rev. A 13 (1976) 2289-2298.

4) H. Mori, M. Komatsu, K. Takeda and H. Fujita: Phil. Mag. Lett. 63 (1991) 173-178.

5) H. Yasuda and H. Mori: Z. Phys. D 37 (1996) 181-186.

6) S. Iijima and T. Ichihashi: Jpn. J. Appl. Phys. 24 (1985) 125-128.

7) K. Takayanagi, Y. Tanishiro, K. Kobayashi, K. Akiyama and K. Yagi: Jpn. J. Appl. Phys. 26 (1987) L957-L960.

8) H. Fujita: J. Electr. Microsc. Techn. 3 (1986) 45-56.

9) H. Fujita: Mater. Trans. JIM 31 (1990) 523-537.

10) H. Fujita: Mater. Trans. JIM 35 (1994) 563-575.

11) H. Fujita: J. Electr. Microsc. 48(Suppl) (1999) 983-994.

12) H. Fujita and N. Fujita: Mater. Trans. 42 (2001) 1474-1479.

13) H. Fujita: Mater. Trans. 43 (2002) 1306-1311.

14) For example, K. Kaya and N. Nishii: Cluster, Sangyo Toshio, (1994) Chapter 3, pp. 33-51.

15) D. G. Pettifor and M. A. Ward: Solid State Communications 49 (1984) 291-294.

16) S. Ino: J. Phys. Soc. Jpn. 21 (1966) 346-362.

17) K. Koga: Bulletin of Phys. Soc. Jpn. 59 (2004) 776-780.

18) S. Saito, S. Ohnishi, C. Satoko and S. Sugano: J. Phys. Soc. Jpn. 55 (1986) 1791-1794.

19) R. Uyeda: Progress in Materials Science., Pergamon Press 35 (1991) 196.

20) K. Kimoto and I. Nishida: J. Phys. Soc. Jpn. 22 (1967) 744-756.

21) M. D. Morse: Chem. Rev. 86 (1984) 1049-1109.

22) A. Tokozaki and G. D. Stein: J. Appl. Phys. 49 (1978) 2224-2232. 